\title{
Vertikalverteilung von Nematoden in Beständen nordadriatischer Sargassaceen
}

\author{
JöRG OTT \\ I. Zoologisches Institut der Universität Wien, Wien, Österreich
}

\begin{abstract}
Vertical distribution of nematodes in growths of Sargassaceae in the northern Adriatic. The nematode fauna of Cystoseira-growths in the upper sublittoral of a rocky shore in Yugoslavia shows a stratification in its composition, which varies according to growth-form and exposition. Typical faunas on the stems, the highly branched parts and the free-waving tips were distinguished. Thick epigrowth shows a marked influence on the composition of the nematode fauna. The effects of the factors (1) water movement, (2) sediment content, (3) substrate structure and (4) nutrition are discussed. The decrease of water-movements measured ranges from decimetres outside the growth to millimetres within the epigrowth. A graph of the isokines in a Cystoseira-growth is given. Sediment content is correlated to populationdensity in parts of comparable growth-form. Minute structures of the algae surface are responsible for the possibilities of locomotion; the stable interstitial space within the epigrowth of calcareous red algae favours aberrant types of locomotion. Large forms find suitable conditions in the colonies of Bryozoa growing at the stems. The distribution of feeding-types (WIESER 1953) shows a characteristic pattern with the epigrowth-feeders mainly at the tips of the thalli, predators and deposit-feeders dominating at the bases. A species with a buccal cavity of the predator type, Enoplus meridionalis, seems to change from epigrowth-feeder as a juvenile (living among the tips) to predator as an adult (only to be found at the bases).
\end{abstract}

\section{EINLEITUNG}

Die wissenschaftliche Taucherei hat die Behandlung der komplizierten Benthosprobleme bis ins Detail ermöglicht. Sie ist den früheren Sammelmethoden eindeutig überlegen. Die Taucherei gestattet es, die vorliegende Arbeit über einen Schichtenbau der Nematodenverteilung in Sargassaseenbestände durchzuführen. Die Idee zu dieser Arbeit geht zurïck auf einen Hinweis meines verehrten Lehrers, Professor RIEDL (1953), und auf die Arbeiten von WIEsER (1951, 1952, 1954, 1959) über die algenbewohnende Mikrofauna.

\section{MATERIAL UND METHODIK}

Das Material sammelte ich während eines Frühjahrs- und eines Sommeraufenthalts am Institut za Biologiju Mora in Rovinj, Jugoslawien. Die Arbeiten wurden freitauchend durchgeführt und dabei die Proben den Algenbeständen schichtweise entnom- 
men. Die Nematoden wurden nach Ausschütteln in $4 \%$ igem Formol oder in $\mathrm{MgCl}_{2}-$ Lösung aus dem Bodensatz gewonnen und ihre systematische Zugehörigkeit nach Anfertigung von Dauerpräparaten determiniert. Von den einzelnen Algenregionen bestimmte ich den Adsorptionskoeffizienten nach WIESER (1951); dieser Koeffizient gibt das Gewicht des von der Algenoberfläche und den Kapillaren festgehaltenen Wassers in Prozent des Algengewichtes an und ist ein relatives Maß der Oberfläche. Auf diese Weise lassen sich Individuenzahlen pro g Algengewicht in der Besiedlungsdichte proportionale Zahlen umwandeln. Eine repräsentative Auswahl von Algenbüscheln wurde zur Sedimentbestimmung ausgeschüttelt und das so erhaltene Sediment im trockenen Zustand gewogen. Für die quantitative Ermittlung der Faunenübereinstimmung verwendete ich die von RIEDL (1953) beschriebene Homogenitätsuntersuchung.

Die Methode der Wasserbewegungsmessung wird auf p. 421 behandelt. Ich möchte hier aber bereits Herrn Dr. H. FoRstNeR, der mir seine Meßgeräte, Erfahrung und Zeit zur Verfügung stellte, herzlich danken.

\section{ALGEN UND FAUNA}

\section{Cystoseiraspicata}

Die Proben wurden dichten Beständen an einem stark exponierten Standort - an einem steilen Declivium der Westseite der Insel Katarina - entnommen. Knapp unterhalb der Niedrigwasserlinie bilden reine Bestände von C. spicata und Mischbestände mit C. abrotanifolia einen Gürtel, der bis $0,75 \mathrm{~m}$ unter die Niedrigwasserlinie reicht. Die Dichte der C. spicata-Bestände (durchschnittlich 8 Büschel pro $\mathrm{dm}^{2}$ ) läßt die Kronen lïckenlos aneinanderschließen, so daß auch bei starker Wasserbewegung nur selten der Kontakt $\mathrm{zwischen} \mathrm{ihnen} \mathrm{gelöst} \mathrm{und} \mathrm{der} \mathrm{Untergrund} \mathrm{freigelegt} \mathrm{wird} \mathrm{(Abb.} \mathrm{1,} \mathrm{links).}$
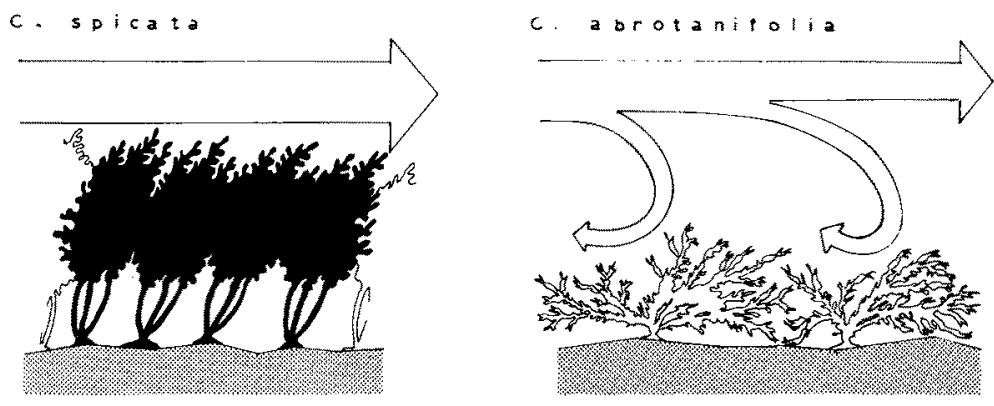

Abb. 1: Wirkung starker Wasserbewegung auf Cystoseirenbestände verschiedener Dichte und Wuchsform

Je 3 Proben wurden folgenden Regionen entnommen: (1) Zweigspitzen, etwa 4 bis $5 \mathrm{~cm}$ lang, mit starkem Diatomeenbewuchs. (2) Eigentliche Kronen mit starker Verzweigung und in Thallusgruben, den Krypten, stehenden Büscheln einreihiger Haare. (3) Rhizoide und Stengel mit gut entwickeltem pflanzlichem und tierischem Auf- 
Tabelle 1

Die Nematodenfauna von Cystoseira spicata. Für jede Art ist Frequenz, die Zahl der gefundenen Individuen und die Dominanz in \% (von links nach rechts) in den einzelnen Regionen angegeben

\begin{tabular}{|c|c|c|c|c|c|c|c|c|}
\hline Cystoseira spicala & Spitzen & & $\mathbf{K r}$ & one & & Bas & & \\
\hline Anticoma acuminata & & & & & & 3 & 47 & 11,3 \\
\hline Trefusia sp. & & & & & & 1 & 1 & 0,2 \\
\hline Oxystomina filiformis & 1 & 0,5 & 1 & 1 & 0,5 & 3 & 10 & 2,4 \\
\hline Phanoderma cocksi & 1 & 0,5 & & & & 3 & 30 & 7,2 \\
\hline Thoracostoma sp. & & & & & & 1 & 1 & 0,2 \\
\hline Enoplus meridionalis & 72 & 34,6 & 3 & 44 & 23,3 & 3 & 110 & 26,7 \\
\hline Paroncbolaimus parapapilliferus & & & & & & 2 & 15 & 3,6 \\
\hline Oncholaimus dujardini & & & 2 & 2 & 1,0 & 3 & 11 & 2,6 \\
\hline Viscosia sp. & & & & & & 2 & 15 & 3,6 \\
\hline Eurystomina ornatum & 2 & 1,4 & 3 & 7 & 3,7 & 3 & 21 & 5,0 \\
\hline Symplocostoma teruicolle & 2 & 1,4 & & & & & & \\
\hline Calyptronema sp. & & & & & & 1 & 1 & 0,2 \\
\hline Syringolaimus striaticaudatus & & & 2 & 3 & 1,6 & & & \\
\hline Cyatholaimus gracilis & 13 & 6,2 & $\overline{3}$ & 9 & 4,8 & 3 & 12 & 2,9 \\
\hline Paracantbonchus micoletzkyi & & & & & & 1 & 1 & 0,2 \\
\hline Monoposthia mediterranea & & & 2 & 3 & 1,6 & 1 & 1 & 0,2 \\
\hline Microlaimus sp. & & & & & & 1 & 1 & 0,2 \\
\hline Acanthopharynx perarmatus & & & & & & 3 & 52 & 12,6 \\
\hline Halichounolaimus clavicauda & & & & & & 2 & 9 & 2,2 \\
\hline Euchromadora striata & 86 & 42,3 & 3 & 55 & 29,2 & 2 & 4 & 0,9 \\
\hline E. mediterranea & & & & & & 3 & 10 & 2,4 \\
\hline Chromadorella membranata & 21 & 10,1 & 2 & 8 & 4,3 & 2 & 9 & 2,2 \\
\hline Ch. parapoecilosoma & & & 3 & 10 & 5,3 & & & \\
\hline Chromadora nudicapitata & & & 2 & 2 & 1,0 & 2 & 4 & 0,9 \\
\hline Chromadorina laeta & 2 & 2,4 & 3 & 36 & 19,1 & 2 & 6 & 1,5 \\
\hline Spilophorella paradoxa & 1 & 0,5 & 1 & 4 & 2,1 & 1 & 1 & 0,2 \\
\hline Neocbromadora sp. & & & $i$ & 1 & 0,5 & & & \\
\hline Hypodontolaimus sp. & & & 1 & 1 & 0,5 & 1 & 1 & 0,2 \\
\hline Araeolaimus sp. & & & & & & 3 & 13 & 3,1 \\
\hline Synonema sp. & & & & & & 3 & 17 & 4,1 \\
\hline Metalinbomoets sp. & & & & & & 1 & 2 & 0,5 \\
\hline Monbystera sp. & 1 & 0,5 & & & & 1 & 1 & 0,2 \\
\hline Arten (Gesamtzahl) & 11 & & & 15 & & & 28 & \\
\hline Individuen (Gesamtzahl) & 207 & & & 186 & & & 306 & \\
\hline Individuen/g Algenfrischgewicht & 0,9 & & & 1,05 & & & 2,6 & \\
\hline
\end{tabular}

wuchs (Pseudolithophyllum expansum, Jania rubens, Peyssonellia squamaria, Fosliella farinosa, der Bryozoe Aetea anguina und der Hydroide Sertularella sp.). Diatomeen sind hier nur spärlich vertreten.

Tabelle 1 zeigt die Nematodenbesiedlung der einzelnen Regionen. Beim Vergleich der beiden Kronenabschnitte fällt zunächst auf, daß die Unterschiede in den Faunen kaum quantitativer Natur sind. Die Artenzahl steigt jedoch von 11 auf 17, die Zahl der Arten mit einer Frequenz größer als 1 von 7 auf 12. Enoplus meridionalis, die häufigste Art auf Cystoseira überhaupt, und Eucbromadora striata dominieren mit 34,6 und $42,3 \%$, die dritte, häufiger vertretene Art ist Chromadorella membranata mit $10,1 \%$. Alle 3 Arten sind von einer kräftigen Cuticula bedeckt, die sie gegen die hohe mechanische Beanspruchung in dieser Region schützen kann. An die Stelle von Chroma- 
dorella membranata tritt in dem folgenden Kronenabschnitt die kleinere, zartere Chromadorina laeta mit 19,1\%, die in ihrer Bewegungsweise durch die Härchenbüschel begünstigt ist. Enoplus meridionalis und Euchromadora striata sind auch hier mit 23,3 und $29,2 \%$ die häufigsten Arten. Große Unterschiede zu den beiden Kronenabschnitten

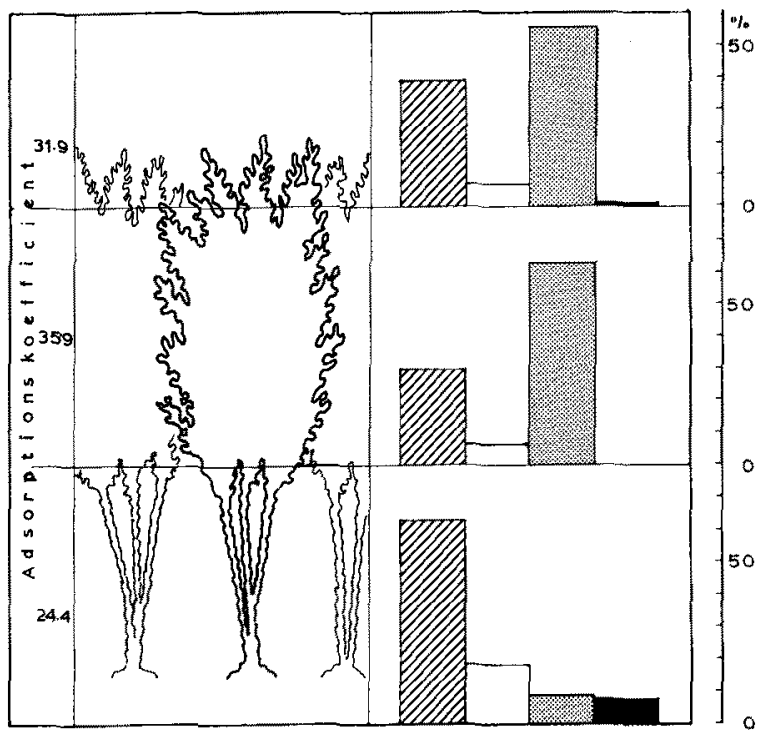

Abb. 2: Anteil der Systemgruppen Enoploidea (gestreif), Cyatbolaimidae und Desmodoridae (weiß), Chromadoridae (punktiert) und Axonolaimoidea und Monbysteroidea (schwarz) an der Nematodenfauna der einzelnen Regionen von Cystoseira spicata

zeigt die Stengelregion. Die Artenzahl steigt auf 28; davon weisen 18 Frequenzen größer als 1 auf. Der Anteil der Enoploidea an der Faunenzusammensetzung steigt auf 63,2\% gegenüber 38,4 und 30,2\% in den Regionen 1 und 2 (Abb. 2). Enoplus meridionalis, Phanoderma cocksi und Anticoma acuminata dominieren mit 26,7, 7,2 und 11,2 Prozent. Den Hauptanteil der Chromadoroidea stellen hier die Cyatholaimidae und Desmodoridae. Chromadoridae treten mit $8,4 \%$ zurück, wobei das fast vollständige Fehlen von Euchromadora striata, die von $E$. mediterania abgelöst wird, auffällt. Dieser Schichtenbau der Fauna - die Homogenität innerhalb der Strata und die Faunengefälle gegeneinander - zeigt sich deutlich in der quantitativen Darstellung der Ubereinstimmungswerte (Abb. 3). Die in allen Teilen so hohe Dominanz von Enoplus meridionalis läßt sich bei Beachtung der Verteilung von adulten und juvenilen Stadien auflösen:

\begin{tabular}{|ccc|}
\hline Region & adult & juvenil \\
\hline Spitzen & - & 72 \\
Mitte & 1 & 43 \\
Basis & 59 & 51 \\
\hline
\end{tabular}



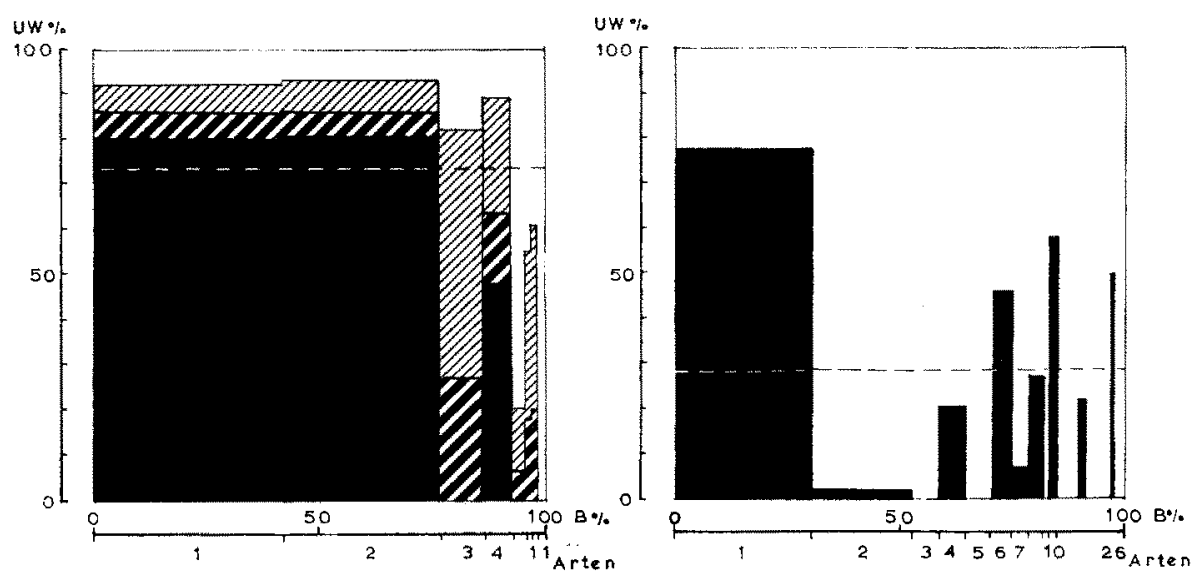

Abb. 3. Homogenitätsdiagramme. Links die Gegenüberstellung dreier Proben aus der Spitzenregion mit Angabe der minimalen, mittleren und maximalen Obereinstimmung, rechts der Vergleich von Spitzen- und Basisregion von Cystoseira spicata. Die gerissene Linie gibt den Grad der Homogenität in $\%$ an

Diese Verteilung kann edaphische, von der Körpergröße abhängige Gründe haben, doch gibt es auch Hinweise auf eine trophische Beziehung.

\section{Cystoseira abrotanifolia an stark exponiertem Standort}

Die Probenentnahmestelle ist identisch mit der von C. spicata. C. abrotanifolia steht hier nahe der Niedrigwasserlinie in kleinen Gruppen in den dichten C. spicataBeständen. Auffallend ist die geringe Dichte der Bestände (etwa 3 bis 4 Büschel pro dm²). Die Verzweigung im Kronenabschnitt ist zwar sehr dicht und der Besatz mit den typischen Härchenbüscheln vorhanden (Adsorptionskoeffizient 36,3), die Zweige sind jedoch weich und ohne Dornästchen. So werden durch die Brandung häufig die Kronen auseinandergelegt, so daß die Büschel bis in substratnahe Teile von der ungeminderten Außenströmung durchsetzt werden (Abb. 1, rechts). Daher besteht die Fauna der oberen Abschnitte nur aus Tieren mit großem Haftvermögen, überwiegend Harpacticiden.

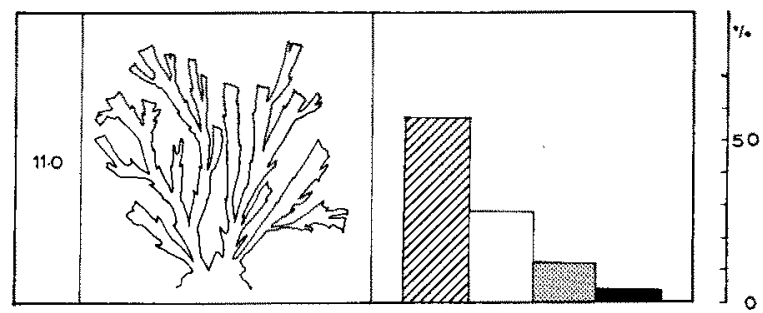

Abb. 4: Anteil der Systemgruppen an der Nematodenfauna der Basen von Cystoseira abrotanifolia des stark exponierten Standortes (vgl. Abb. 2) 
Tabelle 2

Die Nematodenfauna der Basen von Cystoseira abrotanifolia am stark exponierten Standort

\begin{tabular}{|c|c|c|c|}
\hline \multirow{2}{*}{$\begin{array}{l}\text { Cystoseira abrotanifolia } \\
\text { Phanoderma cocksi }\end{array}$} & \multicolumn{2}{|c|}{ Basis } & \multirow[b]{2}{*}{18,2} \\
\hline & 4 & 57 & \\
\hline Thoracostoma sp. & 1 & 1 & 0,3 \\
\hline Enoplus meridionalis & 4 & 95 & 30,4 \\
\hline Paroncholaimus parapapilliferus & 3 & 9 & 2,9 \\
\hline Viscosia sp. & 3 & 6 & 1,9 \\
\hline Etrystomina ornatum & 2 & 3 & 0,9 \\
\hline Syringolaimus striaticaudatus & 3 & 8 & 2,6 \\
\hline Cyatbolaimus gracilis & 4 & 14 & 4,5 \\
\hline Halichoanolaimus clavicauda & 2 & 4 & 1,3 \\
\hline Monoposthia mediterranea & 1 & 4 & 1,3 \\
\hline Acanthopharynx perarmatus & 4 & 65 & 20,8 \\
\hline Euchromadora striata & 2 & 4 & 1,3 \\
\hline E. mediterranea & 2 & 11 & 3,5 \\
\hline Chromadora nudicapitata & 2 & 2 & 0,6 \\
\hline Chromadorina laeta & 3 & 20 & 6,4 \\
\hline Rhabdogaster sp. & 3 & 10 & 3,2 \\
\hline Araeolaimoides sp. & 1 & 1 & 0,3 \\
\hline Arten & \multicolumn{2}{|r|}{17} & \\
\hline Individuen & \multicolumn{2}{|r|}{313} & \\
\hline Individuen/g Algenfrischgewicht & \multicolumn{2}{|r|}{1,1} & \\
\hline
\end{tabular}

Nematoden sind praktisch nicht vertreten (4 Individuen pro $950 \mathrm{~g}$ Algenfrischgewicht). Die basalen Teile bestehen aus einer großen Anzahl von Stengeln, die einer gemeinsamen Basalscheibe entspringen und sehr kurz und bandförmig abgeflacht sind. Die hier vorhandene Nematodenbesiedlung ist weniger dicht als bei C. spicata in der entsprechenden Region (1,1 und 2,6 Individuen pro g Algenfrischgewicht). Tabelle 2 zeigt die Nematodenbesiedlung dieser Region. Die Zahl der Arten bleibt mit 18 hinter der Artenzahl an den C. spicata-Basen zurück. Die häufigsten Arten sind hier Enoplus meridionalis mit 30,4, Phanoderma cocksi mit 18,2 und Acantopharynx perarmatus mit 20,8\%. Auffallend ist, daß Anticoma acuminata hier vollkommen fehlt. Nach der Verteilung dieser Art in den Proben liegt die Vermutung nahe, daß es sich um eine gegen starke Wasserbewegung empfindliche Art handelt.

\section{Cystoseira abrotanifolia a mäßig exponiertem Standort}

Die Proben wurden im Val di Leso, einer durch eine vorgelagerte Insel etwas geschützten Bucht mit flach abfallendem Hartboden, aus dichten und artenreichen Phytalbeständen entnommen. Aus folgenden Regionen wurden gesammelt: (1) Kronen, die dicht mit den in der Ordnung Laminariales häufigen Haarbüscheln besetzt sind (3 Proben). (2) Basalscheibe mit den untersten Stengelabschnitten (2 Proben). Die Zusammensetzung der Fauna zeigt Tabelle 3. Die Kronen enthalten mit 8 Arten bei 168 Individuen eine erstaunlich geringe Artenzahl und sind sowohl quantitativ als auch qualitativ sehr verschieden. Nur 2 Arten sind in allen 3 Proben vertreten (Euchromadora 
Tabelle 3

Die Nematodenfauna von Cystoseira abrotanifolia am mäßig exponierten Standort

\begin{tabular}{|c|c|c|c|c|c|c|}
\hline \multirow{2}{*}{$\frac{\text { Cystoseira abrotanifolia }}{\text { Anticoma acuminata }}$} & \multicolumn{2}{|c|}{ Krone } & \multirow[b]{2}{*}{1,2} & \multicolumn{2}{|c|}{ Basis } & \multirow[b]{2}{*}{8,8} \\
\hline & 2 & 2 & & 2 & 21 & \\
\hline Phanoderma cocksi & & & & 2 & 16 & 6,7 \\
\hline Nasinema sp. & & & & 1 & 1 & 0,4 \\
\hline Thoracostoma sp. & & & & 2 & 14 & 5,9 \\
\hline Enoplus meridionalis & 2 & 3 & 1,8 & 2 & 123 & 51,7 \\
\hline Eurystomina ornatum & 1 & 1 & 0,6 & 2 & 5 & 2,1 \\
\hline Calyptronema sp. & 1 & 2 & 1,2 & 1 & 6 & 2,5 \\
\hline Cyatbolaimus gracilis & & & & 2 & 15 & 6,3 \\
\hline Monoposthia mediterranea & & & & 2 & 4 & 1,7 \\
\hline Euchromadora striata & 3 & 61 & 36,3 & $\hat{1}$ & 5 & 2,1 \\
\hline Euchromadora sp. & 2 & 14 & 8,3 & & & \\
\hline Chromadorella parapoecilosoma & & & & 1 & 2 & 0,8 \\
\hline Chromadora nudicapitata & 3 & 82 & 48,8 & 1 & 4 & 1,7 \\
\hline Chromadorina laeta & 2 & 3 & 1,8 & 2 & 11 & 4,6 \\
\hline Spilophorella paradoxa & & & & 1 & 5 & 2,1 \\
\hline Araeolatmus sp. & & & & 1 & 2 & 0,8 \\
\hline Metalinbomoeus sp. & & & & 1 & 1 & 0,4 \\
\hline Monbystera sp. & & & & 1 & 1 & 0,4 \\
\hline Theristus sp. & & & & 2 & 4 & 1,7 \\
\hline Arten & & 8 & & & 18 & \\
\hline Individuen & & 167 & & & 240 & \\
\hline Individuen / g Algenfrischgewicht & & 1,3 & & & 2,0 & \\
\hline
\end{tabular}

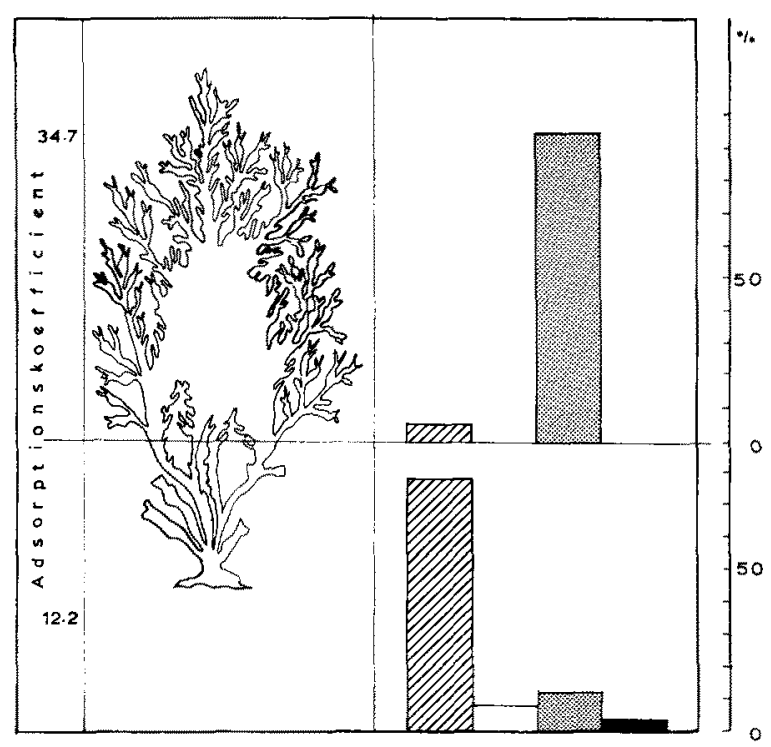

Abb. 5: Anteil der Systemgruppen an der Nematodenfauna der Kronen- und Basisregion von Cystoseira abrotanifolia des mäBig exponierten Standortes (vgl. Abb. 2) 
striata und Chromadorina nudicapitata); die Dominanz der letzteren Art ist auf ihre Häufigkeit in einer einzigen Probe zurückzuführen. Da die Aufsammlungsbedingungen für alle 3 Proben die gleichen waren, sind es möglicherweise mikrotopographische Unterschiede, die in dem stark durch Spalten zerlegten Aufsammlungsort uneinheitliche Expositionsverhältnisse schaffen. Die Basen zeigen ein weit einheitlicheres Bild (8 von 18 Arten sind in beiden Proben vertreten). Da Anderungen der äußeren Bedingungen sich auf die klimatischen Verhältnisse dieser Region schwächer auswirken, ist dies verständlich. Enoplus meridionals dominiert mit 51,7\% stärker als in den anderen Proben. Anticoma acuminata folgt mit $8,8 \%$, die großen Formen Pbanoderma cocksi und Thoracostoma sp. sind mit 6,4 und 5,8\% vertreten. Der Gesamtanteil an Enoploidea ist mit $78,1 \%$ sehr hoch (Abb. 6).

\section{Cystoseira adriatica mit Auf wuchs von Jania rubens}

Die Probe wurde einem mäßig dichten Bestand an einem exponierten Kap (Pt. Barabiga) mit waagerecht abfallenden Felsplatten entnommen. Zur Zeit der Aufsammlung (August) waren die meisten Cystoseiren bis auf die obersten Zweigspitzen dicht mit der Rotalge Jania rubens bewachsen (Abb. 7). Die geänderten Raumstruktur-

Tabelle 4

Die Nematodenfauna des Epiphytenbewuchses auf Cystoseira adriatica. Die erste Spalte gibt die Individuenzahlen, die zweite die Dominanzwerte in $\%$ an

\begin{tabular}{|lrr|}
\hline Cystoseira adriatica & \multicolumn{2}{c|}{ Epiphyten } \\
\hline Anticoma acuminata & 29 & 4,8 \\
Pbanoderma cocksi & 56 & 9,3 \\
Thoracostoma sp. & 4 & 0,7 \\
Enoplus meridionalis & 62 & 10,3 \\
Paroncholaimus parapapilliferus & 15 & 2,5 \\
Symplocostoma tenuicolle & 3 & 0,5 \\
Calyptronema sp. & 10 & 1,7 \\
Syringolaimus striaticaudatus & 3 & 0,5 \\
Cyatbolaimus gracilis & 15 & 2,5 \\
Paracantonchus sp. & 11 & 1,8 \\
Monoposthia mediterranea & 19 & 3,2 \\
Acanthopharynx perarmatus & 14 & 2,3 \\
Croconema sp. & 3 & 0,9 \\
Eucbromadora striata & 23 & 3,8 \\
Chromadorella parapoecilosoma & 86 & 14,3 \\
Chromadora nudicapitata & 9 & 1,5 \\
Spiliphera dolicbura & 45 & 7,5 \\
Rhabdogaster sp. & 186 & 30,1 \\
Hypodontolaimus sp. & 4 & 0,7 \\
Theristus sp. & 1 & 0,2 \\
Metalinbomoeus sp. & 2 & 0,4 \\
\hline Arten & 21 & \\
\hline Individuen & 600 & \\
\hline Individuen/g Algenfrischgewicht & \multicolumn{2}{|c}{} \\
\hline
\end{tabular}




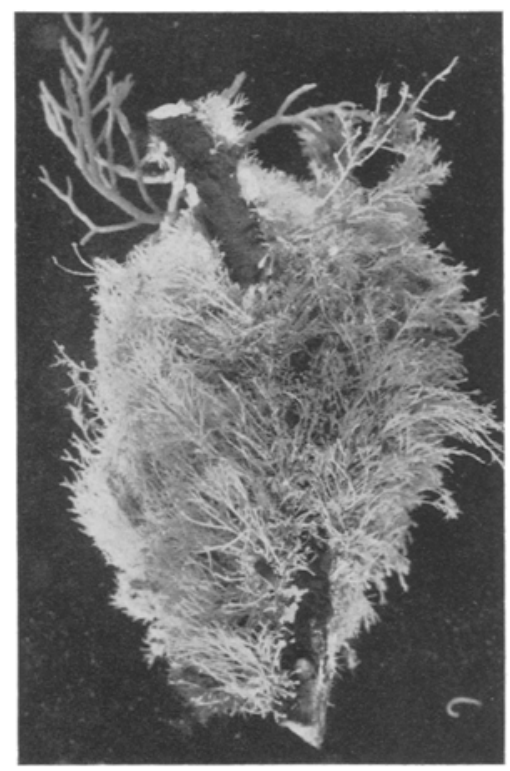

Abb. 6

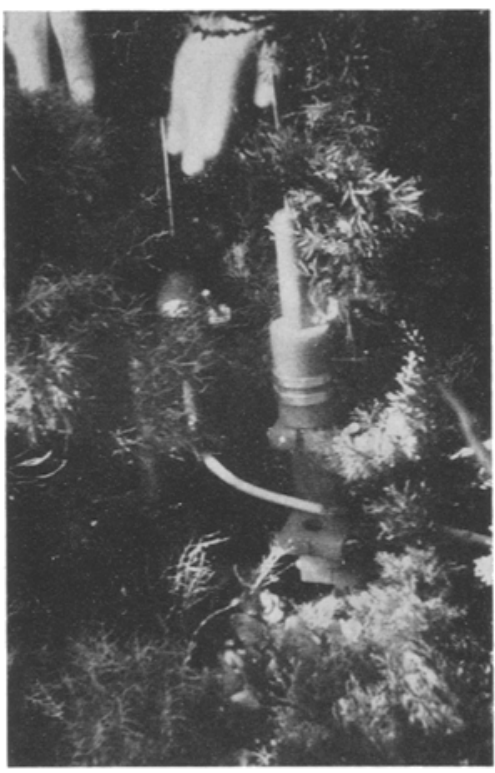

Abb. 7

Abb. 6: Stück eines Stengels von Cystoseira adriatica, bewachsen mit Jania rubens Abb. 7: Einsatz der Thermistormeßsonden in einem Bestand von Cystoseira adriatica. Rechts im Vordergrund ein Meßfühler, dessen Thermistorperle über den Bestand hinausragt, links die Vergleichssonde; die Thermistorperle liegt in etwa $5 \mathrm{~mm}$ Abstand vom Boden des Bestandes

und Wasserbewegungsverhältnisse haben bedeutenden Einfluß auf die Fauna. Die Individuenzahl liegt mit 7,9 pro g Algenfrischgewicht sehr hoch. Bei Berücksichtigung des Adsorptionskoeffizienten $(52,8)$ ist die Besiedlungsdichte immer noch etwa $50 \%$ höher als die der individuenreichsten Region einer unbewachsenen Cystoseira. Der Sedimentgehalt ist gering, aber wahrscheinlich hoch genug, so daß das Nahrungsangebot nicht limitierend wirkt. Das Verhältnis von Enoploidea zu Cbromadoridae nimmt eine Mittelstellung zwischen den für Kronen und Basen bisher gefundenen Werten ein. Der Epiphytenbewuchs scheint also Elemente beider Regionen aufzunehmen.

Besonderheiten in der Faunenzusammensetzung erweisen diesen Lebensraum jedoch als durchaus eigenständig. In Tabelle 4 fällt besonders die hohe Dominanz von Rhabdogaster sp., Chromadorella parapoecilosoma und der langschwänzigen Spiliphera dolichura auf. Dagegen fehlen die kleinsten Formen des schlängelnden Bewegungstypus, Chromadorina laeta und Chromadora nudicapitata, fast vollkommen.

\section{DIE UMWELTFAKTOREN}

\section{Wasserbewegung}

Den Einfluß der Wasserbewegung auf die Fauna mariner Algen behandelte WIESER (1959). Die Vergleiche beziehen sich auf Ortlichkeiten verschiedener Exposition, 
Tiefenzonen und Algenwuchsformen. Riedl (1964) untersuchte die Wasserbewegung im Felslitoral und ihre Wirkung auf Sedentarier. Er wies auf die steilen Gefälle der Teilchengeschwindigkeiten hin, die durch Substratgliederung bedingt sind; dies vermutete er auch für dichte Algenbestände und Epiphytenaufwuchs.

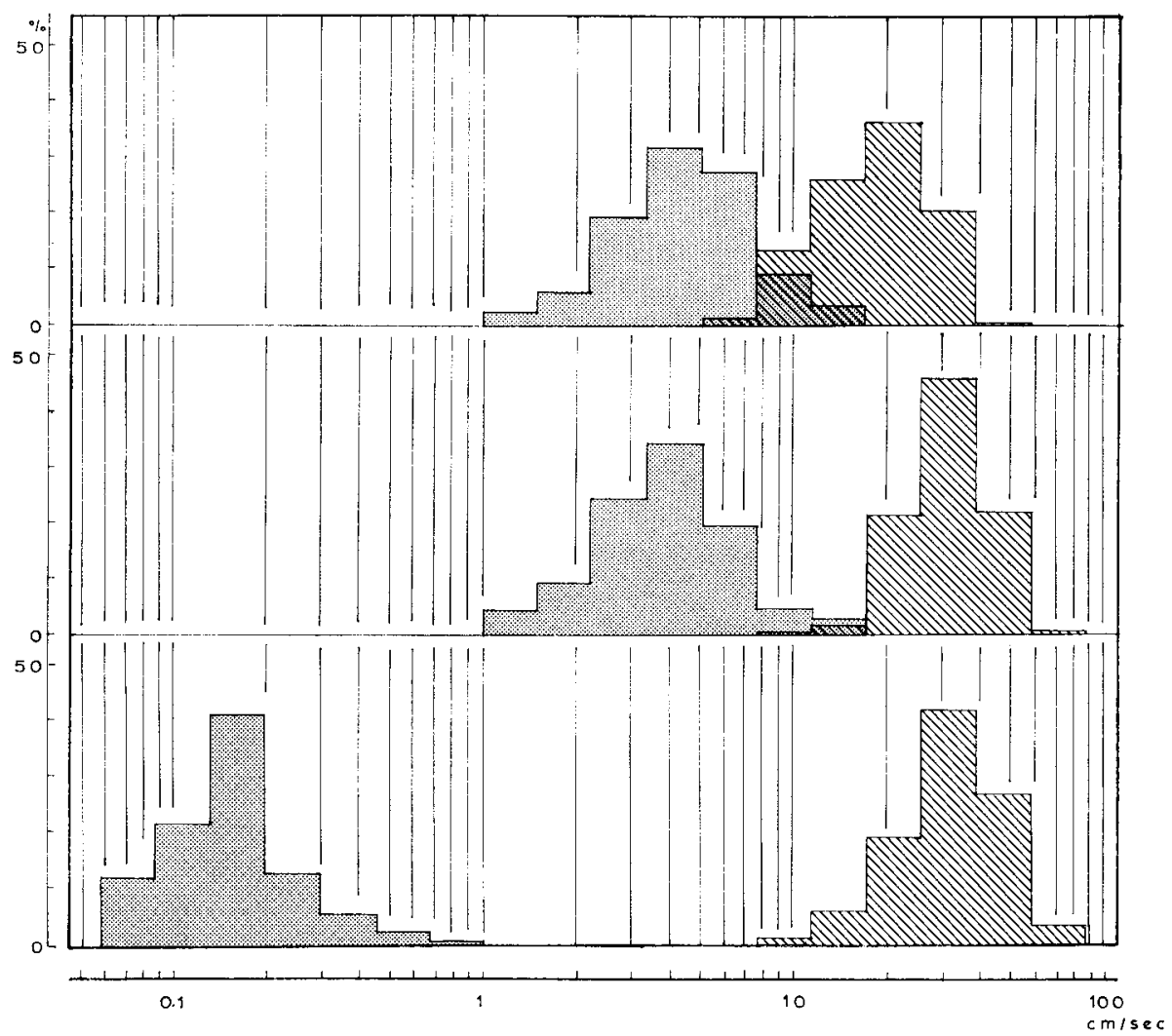

Abb. 8: Diagramm der mittleren Häufigkeit der Wasserbewegungsgeschwindigkeit am Boden eines Cystoseirenbestandes (oben, punktiert), in einem Cystoseirenbiischel (Mitte) und im Inneren des Epiphytenaufwuchses (unten), jeweils im Vergleich zur Wasserbewegungsgeschwindigkeit knapp oberhalb des Bestandes (gestreift). Auf der Abszisse ist die Geschwindigkeit in $\mathrm{cm} / \mathrm{sec}$ in logarithmischem Maßstab, auf der Ordinate die Häufigkeit der einzelnen Klassen in $\%$ linear aufgetragen

Um solche Verhältnisse zu messen, wurde ein Bestand von Cystoseira adriatica an einem exponierten Standort ausgewählt. Die Messung der Wasserbewegungsgeschwindigkeit erfolgte mit geheizten Thermistor-Meßsonden (Forstner \& RüTzLER 1966). Die Meßpunkte lagen (a) etwa $5 \mathrm{~mm}$ über dem Boden innerhalb des Bestandes, (b) in einem Cystoseirenbüschel, (c) im dichten Jania rubens-Aufwuchs jeweils im Vergleich zu der über dem Bestand herrschenden Wasserbewegung. Dazu wurde ein Meßfühler so befestigt, daß die Thermistorperle knapp über den Bestand hinausragte und dazu ein zweiter an der entsprechenden Vergleichsstelle, wobei darauf geachtet wurde, die na- 
türliche Bewegung der Cystoseiren in der Brandung nicht zu beeinflussen. Die Werte registrierte ein Fallbügelschreiber mit 2"-Punkteabstand, den ein Zeitschaltwerk in Abständen von 1,5 Minuten von einer Meßsonde auf die an der Vergleichsstelle umschaltete. Die resultierenden Punktewolken geben die mittlere Häufigkeit der Wasserbewegungsgeschwindigkeit wieder, die in Form von Binominaldiagrammen ausgewertet wurde (Abb. 8). Die gemessenen Geschwindigkeiten umfassen einen Bereich von 3 Dezimalen. Bei den gegebenen Außenbedingungen (Seegang 2 bis 3, Wellenhöhe etwa $20 \mathrm{~cm}$ ) lag das Häufigkeitsmaximum der Geschwindigkeiten über dem Bestand zwischen 10 und $20 \mathrm{~cm}$, am Boden bei 3 bis $4 \mathrm{~cm} / \mathrm{sec}$.

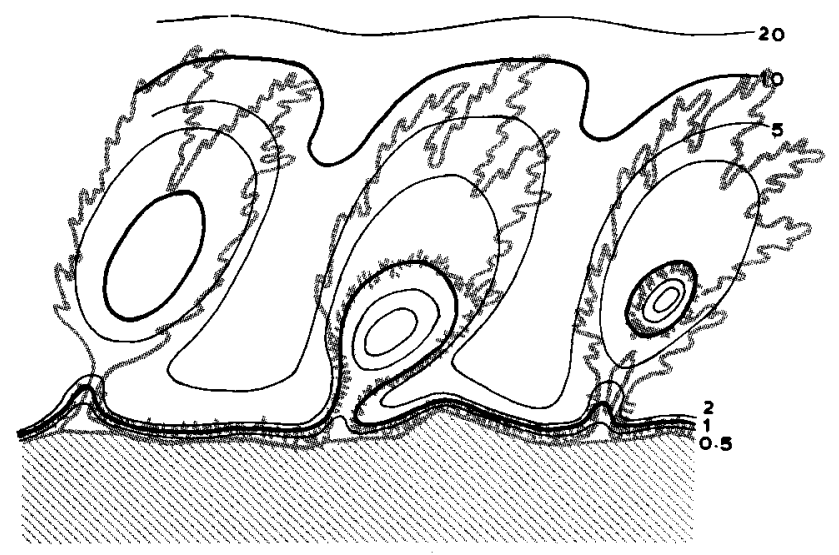

Abb. 9: Schema des Verlaufes der Isokinen in einem Cystoseirenbestand bei einer Wasserbewegungsgeschwindigkeit von $20 \mathrm{~cm}$ pro Sekunde über dem Bestand. Die Beispiele zeigen (von links nach rechts) ein unbewachsenes, ein stark und ein schwachbewachsenes Algenexemplar

Bei größeren Außengeschwindigkeiten (Häufigkeitsmaximum 20 bis $30 \mathrm{~cm}$ ) lagen die Werte innerhalb des Algenbüschels in der Hauptsache ebenfalls zwischen 3 und $4 \mathrm{~cm}$. Das stärkste Gefälle ergab sich erwartungsgemäß gegen das Innere des dichten Aufwuchses, dessen größte Strömungshäufigkeit bei 1 bis $2 \mathrm{~mm}$ pro Sekunde lag und in dem Geschwindigkeiten von $8 \mathrm{~mm}$ nicht überschritten wurden. Den großen Einfluß, den dies auf die Besiedlungsmöglichkeit für Nematoden ausübt, läßt die hohe Besiedlungsdichte erkennen. Abbildung 9 bringt den Entwurf eines Isokinendiagrammes in einem Cystoseirenbestand, das die Hauptgefälle der Wasserbewegungsgeschwindigkeiten verdeutlichen soll.

\section{Sedimentgehalt}

Die Wirkung des Sedimentgehaltes - ich verwende den Begriff Sediment hier im Sinne von WIESER (1954) (alles lose organische und anorganische Material, das an den Algen abgelagert ist) - auf die Mikrofauna des Phytals behandeln DAHL (1948) und für Nematoden im besonderen WIESER $(1959,1951,1954)$. WIESER unterscheidet als Wir- 
kungen Veränderungen der mechanischen und trophischen Struktur des Lebensraumes. Die Reaktionen der Tierwelt können nun quantitativer und qualitativer Natur sein.

Abbildung 10 zeigt den Sedimentgehalt der einzelnen untersuchten Objekte im Zusammenhang mit der mittleren Individuenzahl pro g Algenfrischgewicht der entsprechenden Region. Es wurden nur die Stengelabschnitte berücksichtigt. In den Kronenabschnitten würde die Masse der sich bei dem Versuch, das Sediment auszuschütteln, loslösenden Haarbüschel und Diatomeen das äußerst spärliche Sediment bei weitem überwiegen. Dies entspricht in gewisser Hinsicht den tatsächlichen Bedingungen, da jene

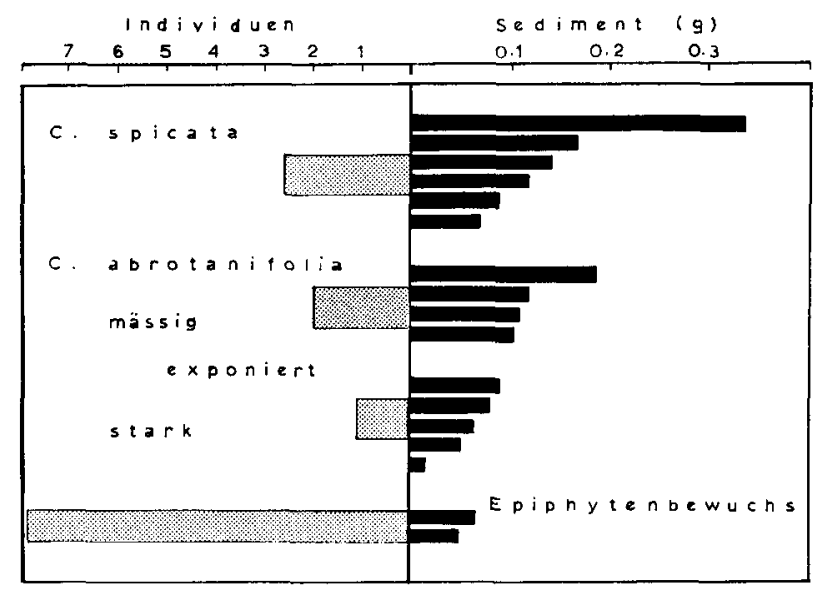

Abb. 10: Diagramm der Beziehung zwischen Sedimentgehalt und mittlerer Individuenzahl der Basen von Cystoseira spicata, Cystoseira abrotanifolia zweier verschiedener Standorte und des Epiphytenbewuchses. Die Individuenzahlen beziehen sich auf $1 \mathrm{~g}$, der Sedimentgehalt auf je $10 \mathrm{~g}$ Algenfrischgewicht

auch für die strukturellen und trophischen Verhältnisse in diesen Regionen zum überwiegenden Teil verantwortlich sind. Die Rolle, welche die Sedimentmenge für die Nematodenbesiedlung beim Aufschließen eines Lebensraumes spielt, zeigt die Korrelation zur Individuenzahl in den ersten drei Fällen. Im Falle des dichten Epiphytenbewuchses, der im letzten Beispiel für die mechanischen Veränderungen verantwortlich ist, tritt die Bedeutung des Sedimentes deutlich zurück.

Die Reaktion der Tierwelt in qualitativer Hinsicht ist jedoch sicher von der Art des Sedimentes abhängig. Die sehr kleinen und im Bewegungstypus aberranten Epsilonematiden und Desmoscoleciden ziehen Sediment in Form von Sand- und Schellkörnchen vor. Die "spanner"- bzw. „schmetterlingsraupenartige“ Bewegungsweise (STAUFFER 1924) dieser Formen verlangt eine stabile Unterlage und freien Lückenraum, um den aufrecht getragenen Körper bewegen zu können. Epsilonematiden können sich schnell und geradlinig auf Sandkörnchen oder auf dem glatten Boden eines Schälchens bewegen, sind aber hilflos und lokomotionsunfähig, wenn sie in eine Ansammlung von lockerem Detritus geraten. Dies setzt sie in Gegensatz zu den Arten, die den bei Nematoden vorherrschenden schlängelnden Bewegungstypus zeigen und die stark genug sind, sich ihren Weg auch durch Detritus zu bahnen. So finden wir Rhabdogaster sp. in den 
Basen von Cystoseira abrotanifolia, wo das Sediment hauptsächlich aus anorganischen Körnchen besteht. An den Basen von Cystoseira spicata, die eine starke Detritusanlagerung zeigen, war dieser Art nicht vertreten. In Jania rubens dominiert diese Art sogar deutlich, da diese Alge ein für diese Art der Fortbewegung ideales Lückenraumsystem mit großem mechanischem Schutz vereint.

\section{Räumliche Struktur}

Die starke Abhängigkeit der Nematoden von der Raumstruktur und den mechanischen Verhältnissen ihrer Umgebung berücksichtigte WIESER (1951) durch die Einteilung der von ihm untersuchten Algenarten in verschiedene Wuchsformtypen (büschelig, rasig, strauchartig, blattartig). Man muß jedoch im Auge behalten, daß es auf großräumigen Algen die spezielle Oberflächengestaltung ist, die den Haupteinfluß auf die Besiedlung ausübt. Der Grund ist in der geringen Größe und in der sehr substratgebundenen Bewegungsweise der Nematoden zu suchen, von denen sich nur einzelne Formen - und auch diese nur ausnahmsweise - durchs freie Wasser schwimmend fortbewegen. Bei den Cystoseiren, die auch als Substrat für andere Algen und viele Sedentarier dienen, muß auch die gestaltsverändernde Wirkung dieses Aufwuchses berïcksichtigt werden.

In den Kronenabschnitten der Cystoseiren bestimmen die schon mehrfach erwähnten, in Thallusgruben stehenden 3 bis $5 \mathrm{~mm}$ langen Haarbüschel die räumliche Struktur. Sie sind der eigentliche Aufenthaltsort solcher Formen der Mikrofauna, die zu ihrer Bewegung engen Substratkontakt vorziehen. Verdeutlicht wurde das durch das Bestreben von Nematoden, Harpacticiden und Chironomidenlarven, sich im Probenschälchen möglichst schnell in solchen losgerissenen Haarbüscheln zu sammeln. Die Bewegungsweise der Nematoden innerhalb dieser Büschel ist nun keineswegs die von STAUFFER (1924) geschilderte gleitende, sich um Ästchen windende - dazu sind die einzelnen Fäden zu dünn und weich. Die Lokomotion entspricht eher einer verlangsamten Schwimmbewegung. Die dicht aneinanderschließenden Fäden, die nur in ihrer Gesamtheit Widerstand bieten und diesen über den ganzen Körper gleichmäßig verteilen, wirken hier wie ein zähes Medium. In Ruhestellung liegen die Tiere parallel der Richtung der Fäden, mit dem Schwanz im basalen Teil des Büschels festgeheftet. Eine Bewegungsweise, wie sie STAUFFER für Algenformen beschreibt, zeigen in dieser Region nur die langen, schlanken Enopliden Oxystomatina filiformis, Eurystomina ornatum und Symplocostoma sp. Sie sind lang genug, um die noch in den letzten Verzweigungen mit etwa $0,5 \mathrm{~mm}$ sehr dicken Cystoseirenästchen mehrmals umschlingen zu können, und finden so genügend Halt.

Die Stengelabschnitte sind in ihren räumlichen Verhältnissen einerseits durch die Sedimentlage, andererseits durch den Aufwuchs bestimmt. Die schuppenförmige Wuchsform von Pseudolithophyllum expansum, das die Basalscheiben fast vollständig bedeckt, begünstigt die Ablagerung von Sediment unter den freien Rändern des Thallus und schafft feine Spalträume. Die anschließende Stengelregion ist bei Cystoseira spicata von Aetea anguina bewachsen, einer Bryozoe mit kriechendem Zoarium, an dem im Abstand von 0,8 bis $1 \mathrm{~mm}$ etwa $1 \mathrm{~mm}$ hohe Zooide aufragen. Durch mehrfaches Öber- 
wachsen der eigenen Zoarien bildet sie eine räumliche Gitterstruktur mit etwa 300 bis $400 \mu$ Maschenweite. Hier bewegen sich die großen Formen Enoplus meridionalis, Phanoderma cocksi, Eurystomina ornatum und Oncholaimiden äußerst schnell und zielsicher, indem sie ihren langen, schlanken Körper in mehreren Windungen durch dieses Gitter „flechten“. Entsprechend verhalten sie sich auch in Probenschälchen, wo sie sich blitzschnell in das Nylongewebe der Netzchen, die dem Verdichten der Proben dienen, schlingen und sich dort parallel einer Fadenrichtung mit hoher Geschwindigkeit bewegen. Dieses Verhalten ist bei den Charakterformen der Kronenabschnitte nie festzustellen. Im Netzchen, das eine Maschenweite von $60 \mu$ besaß, ließ sich auch die bevorzugte Entfernung abschätzen. Die Tiere legten ihre Windungen meist über 5 bis 6 Zwischenräume, was den in Aeteabeständen gemessenen Werten entspricht.

Der im Sommer stark auftretende pflanzliche Aufwuchs hat weitgehende Änderungen zur Folge. In dem hier behandelten Fall handelt es sich um Jania rubens, eine Rotalge mit verkalktem Thallus. So sind die einzelnen Ästchen bei geringer Dicke (Durchmesser etwa $100 \mu$ ) steif genug, um ein wohlausgebildetes, stabiles Lückenraumsystem zu bieten. Die Wirkung auf Epsilonematiden und Desmoscoleciden wurde schon im vorigen Kapitel dargestellt. Das Lückenraumspektrum ist so groß, daß auch die groBen Formen Phanoderma, Thoracostoma, Paroncholaimus und Enoplus günstige Lokomotionsbedingungen vorfinden. Die hier so häufige Chromadorella parapoecilosoma und die langschwänzige Spiliphera gracilicauda können sich nun um die Algenästchen windend fortbewegen. Die kleinsten Formen des schlängelnden Bewegungstypus, denen auch diese Ästchen zu dick wären, sind hier nur schwach vertreten.

GERLACH (1954) stellte für die Nematodenfauna des Sandstrandes Lebensformtypen nach der Bewegungsweise auf. Die vorliegenden Beobachtungen zeigen, daß eine ähnliche Einteilung auch für die Phytalformen der Nematoden sinnvoll wäre. Weitere Beobachtungen sollen dies ermöglichen.

\section{Nahrungsangebot}

Eine Analyse und Deutung der festgestellten Verteilung der Nematodenfauna soll auch trophische Gesichtspunkte einbeziehen. Dies stößt auf eine prinzipielle Schwierigkeit: Über die Ernährung mariner Nematoden liegen nur vereinzelte Angaben vor. Wieser (1953) faßte alle diese Einzelbeobachtungen zusammen und stellte 4 Ernährungstypen auf: Formen ohne eigentliche Mundhöhle (Gruppe 1-A), die als selektive Sinkstofffresser, Formen mit vorhandener, jedoch unbewaffneter Mundhöhle (Gruppe 1-B), die als unselektive Sinkstofffresser betrachtet werden. Formen mit kleiner Mundhöhlenbewaffnung (2-A), die wahrscheinlich Aufwuchsfresser sind, und solche, deren starke Mundhöhlenbewaffnung (2-B) auf räuberische Ernährung schließen läßt.

Die Verteilung dieser Ernährungstypen zeigt Abbildung 11. Sie entspricht, insgesamt gesehen, den Verhältnissen, die von WIESER für exponiertes Phytal angegeben werden. Eine Ausnahme bildet wieder der Jania rubens-Bewuchs, der, durch das Massenvorkommen von Rhabdoagster sp., einen viel höheren Anteil der Gruppe 1-A, aufweist. Daß dafür weniger trophische als edaphische Verhältnisse als Grund angenommen werden, wurde schon erläutert. Allgemein dominieren in den Kronenabschnitten 
die Aufwuchsfresser des Typs 2-A. Ebenso ist die Gruppe 2-B gut vertreten. Die Sedimentfresser der Gruppen 1-A und B fehlen fast vollständig. Das entspricht der Verteilung der für die Gruppen angenommenen Nahrung. Die oberen Abschnitte sind ja praktisch sedimentfrei, jedoch reich an Diatomeenaufwuchs. In den Basisabschnitten steigt, dem Steigen des Sedimentgehaltes entsprechend, der Anteil an Formen des Typs 1-A; die Aufwuchsfresser treten zugunsten dieser Formen und der Räuber zurück.

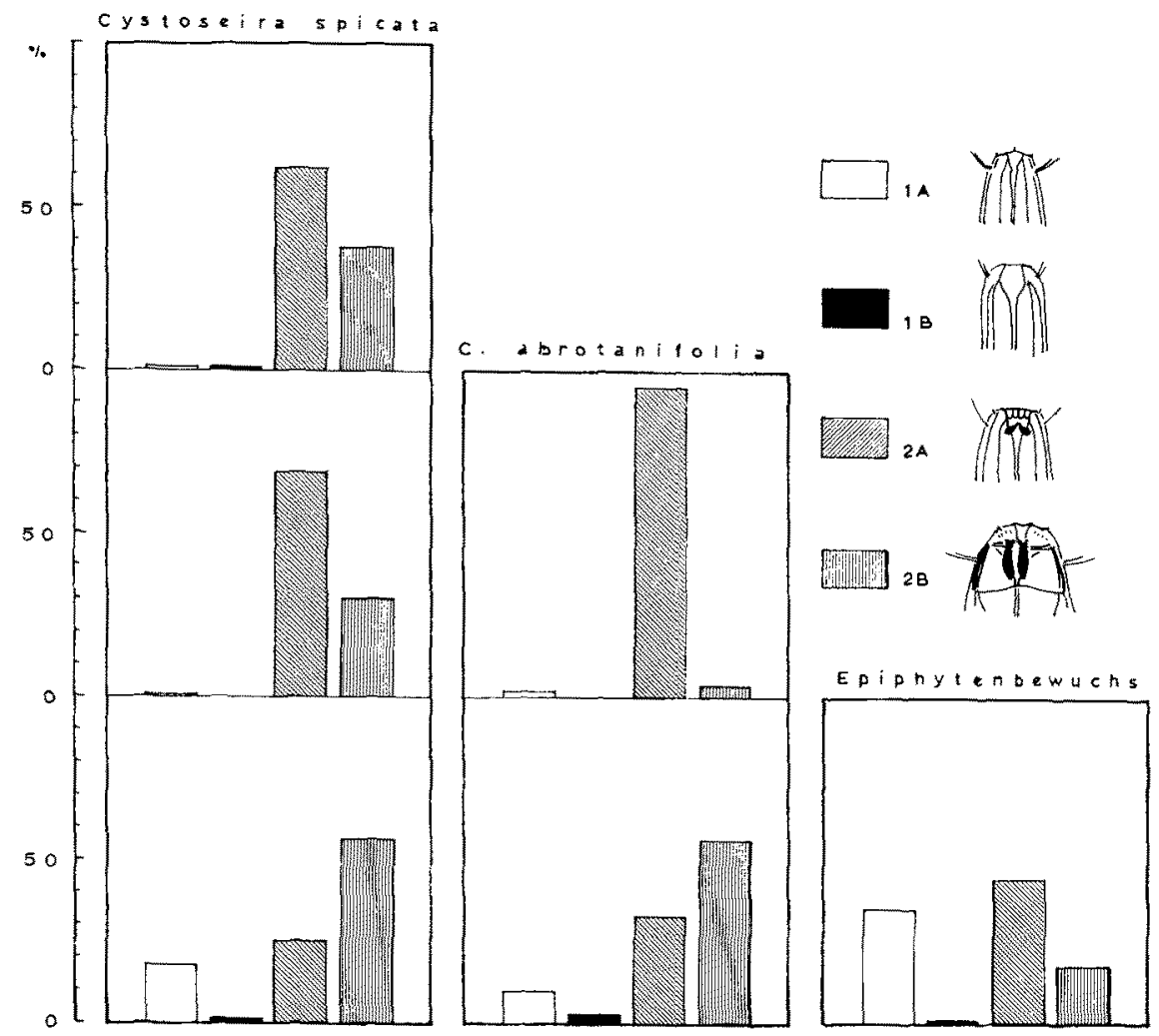

Abb. 11: Verteilung der Mundhöhlentypen (nach WIESER 1953) auf Spitzen-, Kronen- und Basisregion von Cystoseira spicata (links, von oben nach unten), auf $K$ rone und Basis von Cystoseira abrotanifolia (Mitte, von oben nach unten) und im Epiphytenbewuchs

Aus meinem (in dieser Hinsicht noch nicht vollständig durchgearbeiteten) Material möchte ich nur zwei Beobachtungen, die größeres Interesse verdienen, herausgreifen: Im Darm von Paroncholaimus parapapilliferus fand ich sehr regelmäßig die Borsten von Sabelliden. Die größte Häufigkeit und das Auftreten geschlechtsreifer Individuen dieses Nematoden gingen nun parallel dem Massenauftreten solcher Polychäten im Epiphytenaufwuchs. Die auffällige Verteilung der Entwicklungsstadien von Enoplus meridionalis innerhalb der Cystoseirenbestände wurde schon erwähnt. Nun konnte ich in einigen jungen Stadien Diatomeenschalen im Darm beobachten, in älteren und adul- 
ten hingegen nie. Adulte hatten in manchen Fällen Reste anderer Nematoden - in einem Fall auch Polychaetenborsten - im Darm. Es liegt die Vermutung nahe, daß Enoplus meridionalis seine ersten Entwicklungsstadien als Aufwuchsfresser in den Kronen durchlebt, dann diese „Kinderstube“ verläßt und in der Basisregion als Räuber weiter existiert.

\section{ZUSAMMENFASSUNG}

1. Im obersten Sublitoral wurden an verschieden exponierten Küstenpunkten aus Beständen von drei Cystoseira-Arten unterschiedlicher Wuchsform Proben verschiedener Thallusregionen entnommen und die Nematodenfauna untersucht.

2. Je nach Wuchsform des Thallus lassen sich typische Faunenzusammensetzungen für Spitzen-, Kronen- und Basisregion unterscheiden.

3. Der Aufwuchs von Jania rubens ist durch hohe Besiedlungsdichte und die Häufigkeit von Rhabdogaster sp. gekennzeichnet.

4. Die Wasserbewegungsgeschwindigkeiten wurden am Boden des Bestandes, in der Krone eines Cystoseirenexemplares und im Epiphytenbewuchs gemessen. Ein Schema eines Ausschnittes eines Cystoseirenbestandes zeigt den Verlauf der Isokinen in einer Bewegungsphase.

5. Der Sedimentgehalt der Algen erweist sich der Besiedlungsdichte korreliert. Eine Ausnahme bildet der Epiphytenbewuchs, der eine höhere Besiedlungsdichte zeigt.

6. Feinste Oberflächenstrukturen erweisen sich für Lokomotion und Besiedelbarkeit bestimmend. Das relativ stabile Lückenraumsystem von Jania rubens begünstigt aberrante Bewegungstypen (z. B. Rhabdogaster sp.).

7. Die Verteilung der Mundhöhlentypen (nach WIESER 1953) zeigt ein Oberwiegen der Aufwuchsfresser in den Spitzen- und Kronenregionen. In den Basisabschnitten steigt der Anteil an Räubern (bzw. Omnivoren) und Sinkstofffressern. Juvenile Stadien von Enoplus meridionalis, die in der Hauptsache die Kronen bewohnen, ernähren sich offensichtlich von Diatomeen; adulte Individuen hingegen, die nur an den Basen gefunden wurden, sind wahrscheinlich Räuber.

\section{ZITIERTE LITERATUR}

DAHL, E., 1948. On the smaller arthropoda of marine algae, especially in the polyhaline waters off the Swedish west coast. Unders. Öresund 35, 1-193.

Forstner, H. \& Rützler, K., 1966. Probleme und Methoden mikroklimatischer Messungen im Litoral (im Druck).

Gerlach, S. A., 1954. Die Nematodenbesiedlung des Sandstrandes und des Küstengrundwassers an der italienischen Küste. 2. Okologischer Teil. Archo zool. ital. 39, 311-359.

RIEDL, R., 1953. Quantitativ-ökologische Methoden mariner Turbellarienforschung. Öst. zool. Z. 4, 108-145.

- 1964. Die Erscheinungen der Wasserbewegung und ihre Wirkung auf Sedentarier im mediterranen Felslitoral. Helgoländer wiss. Meeresunters. 10, 155-186.

STAUfFer, H., 1924. Die Lokomotion der Nematoden. Beiträge zur Kausalmorphologie der Fadenwürmer. Zool. Jb. (Syst., Ökol. Geogr. Tiere) 49, 1-118. 
WIESER, W., 1951a. Untersuchungen über die algenbewohnende Mikrofauna mariner Hartböden. 1. Zur Ơkologie und Systematik der Nematodenfauna von Plymouth. Öst. zool. Z. 3, $425-480$.

- 1951b. Über die quantitative Bestimmung der algenbewohnenden Mikrofauna felsiger Meeresküsten. Oikos 3, 124-131.

- 1953. Die Beziehung zwischen Mundhöhlengestalt, Ernährungsweise und Vorkommen bei freilebenden marinen Nematoden. Ark. Zool. (Ser. 2) 4, 439-484.

- 1954. Untersuchungen über die algenbewohnende Mikrofauna mariner Hartböden. 3. Zur Systematik der freilebenden Nematoden des Mittelmeeres... Hydrobiologia 6, 144-217.

- 1959. Zur Okologie der Fauna mariner Algen mit besonderer Berüicksichtigung des Mittelmeeres. Int. Rev. ges. Hydrobiol. 44, 137-180.

\section{Diskussion im Anschluß an den Vortrag Oтт}

ZAvodnIK: Es ist bekannt, daß die Thallus-Form der Cystoseira-Arten mit der Jahreszeit wechselt. Hat dieser Umstand vielleicht einen Einfluß auf die Verteilung der NematodenPopulation?

OTT: Die Beantwortung dieser Frage wird das Ziel weiterer Untersuchungen sein. Die Veränderungen der Fauna im Fall von Jania rubens repräsentieren offenbar erste Hinweise.

Gessner: Die Verhältnisse der Wasserbewegung lassen vermuten, daß auch der Sauerstoffgehalt gegen das Innere der Thalli ein Gefälle zeigen könnte. Wurden solche Untersuchungen durchgeführt?

OTT: Die chemischen Verhältnisse innerhalb der Cystoseirenbestände wurden noch nicht untersucht. Die maximalen Geschwindigkeiten sind jedoch mit mehr als $10 \mathrm{~cm} / \mathrm{sec}$ im Innern eines Cystoseirenbüschels groß genug, um reichlichen Wasserwechsel zu gestatten. 